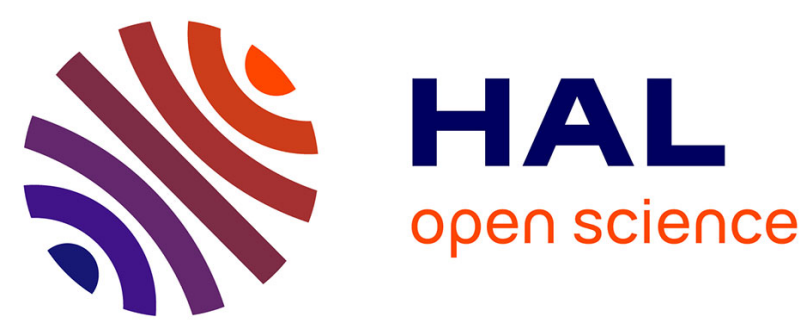

\title{
Isolation and characterization of 28 new microsatellite markers for European flounder (Platichthys flesus L.)
}

Niklas Tysklind, Teresa Neuparth, Gregg R. Ashcroft, Martin I. Taylor, Brett P. Lyons, Ian D. Mccarthy, Gary R. Carvalho

\section{- To cite this version:}

Niklas Tysklind, Teresa Neuparth, Gregg R. Ashcroft, Martin I. Taylor, Brett P. Lyons, et al.. Isolation and characterization of 28 new microsatellite markers for European flounder (Platichthys flesus L.). Molecular Ecology Resources, 2009, 9 (3), pp.1065-1068. 10.1111/j.1755-0998.2009.02589.x . hal02194750

\section{HAL Id: hal-02194750 \\ https://hal.science/hal-02194750}

Submitted on 25 Jul 2019

HAL is a multi-disciplinary open access archive for the deposit and dissemination of scientific research documents, whether they are published or not. The documents may come from teaching and research institutions in France or abroad, or from public or private research centers.
L'archive ouverte pluridisciplinaire HAL, est destinée au dépôt et à la diffusion de documents scientifiques de niveau recherche, publiés ou non, émanant des établissements d'enseignement et de recherche français ou étrangers, des laboratoires publics ou privés. 
PERMANENT GENETIC RESOURCES NOTE

\title{
Isolation and characterization of 28 new microsatellite markers for European flounder (Platichthys flesus L.)
}

\author{
NIKLAS TYSKLIND,* TERESA NEUPARTH,†GREGG R. ASHCROFT, , MARTIN I. TAYLOR, ${ }^{*}$ \\ BRETT P. LYONS, IAN D. MCCARTHY§ and GARY R. CARVALHO* \\ *Molecular Ecology and Fisheries Genetics Laboratory, Environment Centre for Wales, College of Natural Sciences, Deiniol Road, \\ Bangor University, Gwynedd LL57 2UW, UK, +IMAR-Centro de Modelação Ecológica, Faculdade de Ciências e Tecnologia, \\ Universidade Nova de Lisboa, Campus da Caparica, 2829-516 Caparica, Portugal, $\ddagger$ Weymouth CEFAS Laboratory, The Nothe, Barrack \\ Road, Weymouth, Dorset DT4 8UB, UK, §School of Ocean Sciences, College of Natural Sciences, Bangor University, Menai Bridge, \\ Anglesey LL59 5AB, UK
}

\begin{abstract}
The European flounder (Platichthys flesus L.) is used in ecotoxicological studies to provide detailed information on the effects of pollution on individual fish. Data on population and evolutionary level effects are, however, limited. Here, the isolation and characterization of 28 novel species specific microsatellite loci are presented. The number of alleles ranged from 8 to 38 , and observed heterozygosity from 0.542 to 1 .
\end{abstract}

Keywords: biomonitoring, ecotoxicology, flatfish, flounder, microsatellites, population genetics

Received 25 November 2008; revision accepted 15 December 2008

The European flounder (Platichthys flesus L.) is used as a model species in ecotoxicology to examine the ecosystem impacts of anthropogenic pollution. For example, recent studies have examined the effects of toxicants on DNA disruption, gene expression, transcriptomics and tumorigenesis (Stentiford et al. 2003; Marchand et al. 2006; Williams et al. 2008). Although some microsatellite markers have been used on European flounder (Casas et al. 2005; HemmerHansen et al. 2007), only six non-EST-linked species-specific loci have been published to date. Increasing this number will extend the range of applications, such as population assignment, localized impacts of toxicant exposure, and ultimately contribute towards the production of linkage maps. Here we describe the development and characterization of 28 novel microsatellite markers developed from European flounder.

European flounder $(n=2)$ were collected from the Irish Sea $\left(53^{\circ} 18^{\prime} 56.45^{\prime \prime} \mathrm{N} ; 3^{\circ} 53^{\prime} 59.64^{\prime \prime} \mathrm{W}\right)$ and fin clips stored in $100 \%$ ethanol until processing. A microsatellite-enriched genomic library was constructed following the protocol of Tysklind et al. (2009). From this library, 1344 colonies were screened for microsatellites by polymerase chain reaction (PCR) amplification with M13 Forward primer and a

Correspondence: Niklas Tysklind, Fax: 0044 (0) 1248 368131; E-mail: ntysklind@univ.bangor.ac.uk mixture of non-biotinylated microsatellite probes. One hundred and ninety-six positive amplicons were sequenced (Macrogen Inc.), edited, analysed and checked for duplicates using BioEdit (Hall 1999). Enrichment efficiency was high at $87.8 \%$. Primers were designed either side of 47 putative microsatellites using Primer 3 (Rozen \& Skaletsky 2000), and tested for successful amplification at several annealing temperatures on 3\% TBE agarose gels. M13-tailed forward primers were then ordered (Schuelke 2000). Nested PCRs with tailed forward primer, reverse primer, and FAMlabelled M13-tail oligos were used for genotyping. PCR cocktails of $10 \mu \mathrm{L}$ final volume contained around $20 \mathrm{ng}$ of DNA, $1 \times$ GoTaq Flexi buffer (Promega), $1.5 \mathrm{~mm} \mathrm{MgCl}_{2}$, $125 \mu \mathrm{M}$ dNTP, $0.1 \mu \mathrm{M}$ forward-tailed primer, $0.5 \mu \mathrm{M}$ of reverse primer, $0.5 \mu \mathrm{M}$ of FAM-labelled M13 tails, and $0.5 \mathrm{U}$ GoTaq DNA polymerase (Promega). PCRs were carried on a Bio-Rad Tetrad2 Peltier Thermal Cyclers and the thermocycling programmes were as follows: an initial denaturation phase of $3 \mathrm{~min}$ at $95^{\circ} \mathrm{C}$, followed by 13 cycles of $30 \mathrm{~s}$ at $95^{\circ} \mathrm{C}, 45 \mathrm{~s}$ at $55^{\circ} \mathrm{C}, 60 \mathrm{~s}$ at $72{ }^{\circ} \mathrm{C}$, then 31 cycles of $30 \mathrm{~s}$ at $94{ }^{\circ} \mathrm{C}, 45 \mathrm{~s}$ at $50{ }^{\circ} \mathrm{C}, 60 \mathrm{~s}$ at $72^{\circ} \mathrm{C}$, and finishing with a 30 min extension phase at $72{ }^{\circ} \mathrm{C}$.

Two samples of 24 individuals collected from the Mersey Estuary (53 $\left.19^{\prime} 28.70^{\prime \prime} \mathrm{N}, 2^{\circ} 54^{\prime} 39.74^{\prime \prime} \mathrm{W}\right)$ and the Tyne Estuary $\left(54^{\circ} 59^{\prime} 10.21^{\prime \prime} \mathrm{N} .1^{\circ} 27^{\prime} 49.47^{\prime \prime} \mathrm{W}\right)$ were genotyped on an ABI 3130xl Genetic Analyser (Applied Biosystems) with an 
Table 1 Characterization of 28 microsatellite loci isolated from Platichthys flesus in two estuarine populations. Motif, repeat sequence of the isolated clone; $T_{a}$, annealing temperature; $N$, number of individuals successfully amplified (out of 48 ). $N_{\mathrm{a}}$, number of alleles; range, allele size range; $H_{\mathrm{O}}$, observed heterozygosity; $H_{\mathrm{E}}$, expected heterozygosity; $P$, associated probability value of conformation with Hardy-Weinberg equilibrium (HWE). Bold $P$ values indicate significant deviation from HWE after Bonferroni correction

\begin{tabular}{|c|c|c|c|c|c|c|c|c|c|c|c|c|c|c|c|c|}
\hline \multirow[b]{2}{*}{ Name } & \multirow{2}{*}{$\begin{array}{l}\text { Accession } \\
\text { no. }\end{array}$} & \multirow[b]{2}{*}{ Motif } & \multirow[b]{2}{*}{ Forward primer } & \multirow[b]{2}{*}{ Reverse primer } & \multicolumn{4}{|c|}{ Overall } & \multicolumn{4}{|c|}{ Mersey Estuary } & \multicolumn{4}{|c|}{ Tyne Estuary } \\
\hline & & & & & $T_{\mathrm{a}}$ & $N$ & $N_{\mathrm{a}}$ & Range & $N_{\mathrm{a}}$ & $H_{\mathrm{O}}$ & $H_{\mathrm{E}}$ & $P$ & $N_{\mathrm{a}}$ & $H_{\mathrm{O}}$ & $H_{\mathrm{E}}$ & $P$ \\
\hline FLAC1-32 & FJ360492 & $(\mathrm{AC})_{19}$ & GAGAAACCTCCCACAGGTGA & GGGAAATAACGCTGATACGAA & 55 & 48 & 13 & $156-184$ & 9 & 0.833 & 0.829 & 0.381 & 11 & 0.958 & 0.881 & 0.070 \\
\hline FLAC1-47 & FJ360493 & $(\mathrm{AC})_{7}+(\mathrm{AC})_{16}$ & ACGCACATACGAAGCCGTAG & АTTTCTGCCCAGGGATTACC & 55 & 47 & 10 & $242-270$ & 7 & 0.542 & 0.680 & 0.044 & 8 & 0.652 & 0.700 & 0.500 \\
\hline FLAC2-16 & FJ360494 & $(\mathrm{AC})_{15}$ & ССАТСTACCCCCAGAAAACA & CTCCGGGGGAAACTTAAGAG & 55 & 48 & 20 & 156-202 & 17 & 0.917 & 0.869 & 0.431 & 16 & 0.833 & 0.904 & 0.022 \\
\hline FLAC2-18 & FJ360495 & $(\mathrm{AC})_{20}$ & AAAAGCCAATGACCTGTTGC & GCATGCCAGTGAGAACAATG & 55 & 48 & 20 & $216-266$ & 16 & 0.958 & 0.910 & 0.041 & 18 & 0.833 & 0.901 & 0.074 \\
\hline FLAC2-46 & FJ360496 & $(\mathrm{AC})_{16}$ & TGCATGGTTTTAAATACGACAA & GAACAGCAGCCTTTGTTTTTG & 55 & 45 & 16 & $167-209$ & 13 & 0.875 & 0.868 & 0.316 & 14 & 0.810 & 0.892 & 0.129 \\
\hline FLAC3-19 & FJ360497 & $(\mathrm{AC})_{5} \mathrm{AC}(\mathrm{AC})_{17}$ & GAAACCAGGGGGCTCTTTAG & TCTTAGATCCCCCGAATGTG & 55 & 48 & 11 & $221-279$ & 8 & 0.792 & 0.813 & 0.579 & 11 & 0.833 & 0.858 & 0.537 \\
\hline FLAC4-60 & FJ360498 & $(\mathrm{AC})_{22} \mathrm{TC}(\mathrm{AC})_{5}$ & CGCCTCTTGACACACAGAGA & AGCCCTTCTGTTCGAGTTCA & 55 & 48 & 18 & $179-257$ & 13 & 0.833 & 0.859 & 0.877 & 15 & 0.792 & 0.893 & 0.001 \\
\hline FLAC4-67 & FJ360499 & $(\mathrm{AC})_{27}$ & ACCTGGACCAAACACACACA & CCCCACCATGTCAGAACTTA & 55 & 48 & 27 & 175-296 & 19 & 1.000 & 0.914 & 0.613 & 22 & 0.875 & 0.931 & 0.206 \\
\hline FLAC4-69 & FJ360500 & $(\mathrm{AC})_{5}+(\mathrm{AC})_{14}$ & GGGAGAACCTGTCAAACCTG & AACGCAACACGCATACAAAC & 55 & 47 & 23 & $228-300$ & 20 & 0.875 & 0.934 & 0.161 & 19 & 1.000 & 0.919 & 0.995 \\
\hline FLAC4-7 & FJ360501 & $(\mathrm{AC})_{5} \mathrm{AT}(\mathrm{AC})_{3} \mathrm{GC}(\mathrm{AC})_{23}$ & CGATGGCCTTCTCTT'TTTCA & TTTTGATGCCATTTGCATGT & 55 & 47 & 12 & 169-197 & 12 & 0.913 & 0.837 & 0.969 & 10 & 0.792 & 0.806 & 0.470 \\
\hline FLAC4-81 & FJ360502 & $(\mathrm{AC})^{16} \mathrm{AG}(\mathrm{AC})_{4}$ & CAAGGCTCCTGTGACAGCTT & CCAGTAAGAGATCAAACACG & 55 & 48 & 14 & 174-208 & 12 & 0.708 & 0.867 & 0.020 & 12 & 0.625 & 0.850 & 0.006 \\
\hline FLAG1-11 & FJ360503 & $(\mathrm{AG})_{18}$ & GCGAGAGAGGGAGAAAGAAAA & TTCCTATCTGGTTCAGTCCTTCTT & 55 & 48 & 23 & $235-337$ & 18 & 0.833 & 0.914 & 0.021 & 18 & 0.917 & 0.919 & 0.622 \\
\hline FLAG1-81 & FJ360504 & 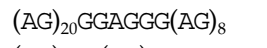 & AAAACCTGGTGGCATATGGT & GCTTTCATTTCCCAGGTCAG & 55 & 47 & 20 & $196-236$ & 17 & 1.000 & 0.899 & 0.688 & 14 & 0.833 & 0.892 & 0.068 \\
\hline FLAG2-76 & FJ360505 & $(\mathrm{AG})_{6} \mathrm{CG}(\mathrm{AG})_{21}$ & АCCTTTCGACCACCTGTCTG & TCTCATGTGCTGCTGTTTCC & 55 & 43 & 25 & $156-226$ & 17 & 0.800 & 0.918 & 0.146 & 21 & 0.870 & 0.928 & 0.034 \\
\hline FLAG3-55 & FJ360506 & $(\mathrm{AG})_{16}$ & GCCAGCTTCATGACACACAC & GCCGATGGCATGTAGAGAAT & 55 & 48 & 13 & $228-266$ & 8 & 0.833 & 0.686 & 0.791 & 11 & 0.833 & 0.797 & 0.064 \\
\hline FLAG3-73 & FJ360507 & $(\mathrm{AG})_{16}$ & TGATGGACAGCTTCAGCATC & GAACACCATCAGGTATCTTCATCA & 55 & 48 & 23 & $257-325$ & 16 & 0.917 & 0.903 & 0.115 & 18 & 0.875 & 0.909 & 0.025 \\
\hline FLAG4-25 & FJ360508 & $(\mathrm{AC})_{15} \mathrm{~A}(\mathrm{AC})_{19}$ & CGGGGTCACAGTTTAACACA & TGTTCATGTGGTTGCATTTG & 55 & 46 & 37 & $120-314$ & 25 & 0.957 & 0.947 & 0.670 & 22 & 0.870 & 0.908 & 0.039 \\
\hline FLAG4-65 & FJ360509 & $(\mathrm{AGAT})_{18}+(\mathrm{AG})_{11}$ & TGTGTGTGAGTGTATGTTTACTTGG & CAGTTGCGCAAGCTAATGTC & 55 & 48 & 24 & $232-302$ & 19 & 0.833 & 0.926 & 0.024 & 22 & 0.958 & 0.941 & 0.506 \\
\hline FLAG4-71 & FJ360510 & $(\mathrm{AG})_{17} \mathrm{AA}(\mathrm{AG})_{5}$ & TGAAAAGGGATAAGAGGGAGA & TTCTAGCTGGACTCAAGGGTAA & 55 & 46 & 30 & $183-247$ & 24 & 0.917 & 0.947 & 0.290 & 20 & 0.955 & 0.934 & 0.546 \\
\hline FLAG5-83 & FJ360511 & $(\mathrm{AGAT})_{16}$ & CCAGTGCAGAGGAGTTTTCAG & TTGAGTTCACTCCTGCACCA & 55 & 41 & 38 & $200-340$ & 28 & 1.000 & 0.957 & 1.000 & 24 & 0.895 & 0.950 & 0.076 \\
\hline FLAG5-87 & FJ360512 & $(\mathrm{AG})_{9} \mathrm{GG}(\mathrm{AG})_{16}$ & TCTTCTCGCTGCATGAACAC & CСGTTTCCTTTGTCCAACAT & 55 & 48 & 23 & $159-263$ & 18 & 1.000 & 0.916 & 0.818 & 20 & 1.000 & 0.917 & 0.017 \\
\hline FLAG6-14 & FJ360513 & $(\mathrm{AG})_{18} \mathrm{AT}(\mathrm{AG})_{8}$ & CAGTAGCAGGGTGTTTTTCCTT & CCAGTAATGACCAAACCCAAA & 55 & 44 & 31 & $226-364$ & 21 & 0.857 & 0.925 & 0.026 & 21 & 0.870 & 0.936 & 0.009 \\
\hline FLAG6-77 & FJ360514 & $(\mathrm{AGAT})_{33}(\mathrm{AT})_{3}(\mathrm{AGAT})_{9}$ & TAAGATAGATAGCTGCATTG & САTGTTTCTTTCACAAATTA & 55 & 48 & 29 & $136-274$ & 23 & 0.833 & 0.944 & 0.043 & 22 & 0.958 & 0.940 & 0.726 \\
\hline FLAG7-17 & FJ360515 & $(\mathrm{AG})_{18}$ & GCAACGAGCTGCTAATTAAGG & CGACCAACACAAACCACTTG & 55 & 48 & 8 & $170-188$ & 6 & 0.917 & 0.759 & 0.251 & 7 & 0.875 & 0.747 & 0.864 \\
\hline FLAG8-19 & FJ360516 & $(\mathrm{AG})_{11} \mathrm{GG}(\mathrm{AG})_{13}$ & ACCTCGGCCAGCACTTAATA & AAAGGGGGCAGATGATTAGG & 55 & 48 & 20 & 165-209 & 16 & 0.875 & 0.901 & 0.555 & 18 & 0.917 & 0.932 & 0.344 \\
\hline FLAG8-37 & FJ360517 & $(\mathrm{GT})_{10} \mathrm{G}(\mathrm{AG})_{8} \mathrm{CG}(\mathrm{AG})_{30}$ & GAACTCCTGTCCTGCTGCTC & CCGTCATCGCTCTCTGAGG & 55 & 47 & 24 & $213-275$ & 16 & 0.826 & 0.872 & 0.299 & 23 & 0.875 & 0.930 & 0.248 \\
\hline FLAG8-41 & FJ360518 & $(\mathrm{AG})_{19}$ & AAATCCAGATGCAGGTCACA & GAGGCTCTGGCTGTTTGTTC & 55 & 48 & 13 & $221-255$ & 11 & 0.917 & 0.783 & 0.068 & 12 & 0.792 & 0.832 & 0.243 \\
\hline FLAG8-89 & FJ360519 & $(\mathrm{AC})_{12}+(\mathrm{AG})_{23}$ & CCCATACAGACAGCTGGTGA & TTTCCCACGATGGAGGAG & 55 & 47 & 23 & $173-223$ & 17 & 1.000 & 0.919 & 0.909 & 20 & 0.875 & 0.915 & 0.402 \\
\hline
\end{tabular}


Table 2 Results of cross-species amplification of 28 microsatellite loci developed from European flounder on other European flatfish species $(n=1 /$ spp.). Amplification conditions identical to those described for European flounder. Presence of microsatellite-like products is indicated with allele sizes, absence with a '-'. Pf, Platichthys flesus; Ll, Limanda limanda; Pp, Pleuronectes platessa; Hp, Hippoglossoides platessoides; Lw, Lepidorhombus whiffiagonis; Sr, Scophthalmus rhombus; Pm, Psetta maxima; Bl, Buglossidium luteum; Ss, Solea solea; Pl, Pegusa lascaris; Mv, Microchirus variegatus; Mk, Microstomus kitt

\begin{tabular}{|c|c|c|c|c|c|c|c|c|c|c|c|c|}
\hline Locus & Pf & $\mathrm{Ll}$ & $\mathrm{Pp}$ & Hp & Lw & $\mathrm{Sr}$ & $\mathrm{Pm}$ & $\mathrm{Bl}$ & Ss & $\mathrm{Pl}$ & $\mathrm{Mv}$ & $\mathrm{Mk}$ \\
\hline FLAC1-32 & $176 / 176$ & $168 / 170$ & $166 / 170$ & $166 / 166$ & - & $166 / 178$ & - & - & $160 / 162$ & - & $154 / 166$ & - \\
\hline FLAC1-47 & $262 / 262$ & - & - & - & - & - & - & - & - & - & - & - \\
\hline FLAC2-16 & $178 / 196$ & $170 / 172$ & $192 / 196$ & $164 / 172$ & $160 / 160$ & $164 / 166$ & $164 / 166$ & $158 / 192$ & $176 / 180$ & $158 / 258$ & - & - \\
\hline FLAC2-18 & $242 / 246$ & $256 / 260$ & $250 / 256$ & $226 / 226$ & - & - & $246 / 248$ & - & $266 / 270$ & $280 / 280$ & $302 / 308$ & $296 / 296$ \\
\hline FLAC2-46 & $187 / 205$ & $179 / 191$ & $181 / 209$ & - & - & - & - & - & $195 / 207$ & - & - & - \\
\hline FLAC3-19 & $221 / 273$ & - & $249 / 249$ & $259 / 263$ & - & $249 / 265$ & $253 / 265$ & - & $251 / 251$ & $267 / 267$ & $237 / 263$ & $267 / 279$ \\
\hline FLAC4-60 & $179 / 183$ & - & - & - & - & - & - & - & - & - & - & - \\
\hline FLAC4-67 & $192 / 199$ & - & - & - & - & - & - & - & - & - & - & - \\
\hline FLAC4-69 & $276 / 285$ & - & & $290 / 292$ & - & - & - & - & $254 / 264$ & $242 / 242$ & - & $246 / 280$ \\
\hline FLAC4-7 & $187 / 189$ & - & $193 / 193$ & - & - & - & - & - & - & - & - & - \\
\hline FLAC4-81 & $174 / 192$ & - & $192 / 198$ & $176 / 188$ & - & - & - & - & - & - & $186 / 194$ & $182 / 192$ \\
\hline FLAG1-11 & $249 / 249$ & - & - & - & - & - & - & - & - & - & - & - \\
\hline FLAG1-81 & $204 / 210$ & - & - & $222 / 222$ & - & $184 / 190$ & - & - & - & - & - & - \\
\hline FLAG2-76 & $170 / 170$ & - & - & - & - & - & - & - & - & - & - & - \\
\hline FLAG3-55 & $256 / 258$ & - & $230 / 236$ & - & - & - & - & - & $228 / 252$ & - & - & - \\
\hline FLAG3-73 & $277 / 295$ & - & - & - & - & - & - & - & - & - & - & - \\
\hline FLAG4-25 & $254 / 284$ & - & - & - & - & - & - & - & - & - & - & - \\
\hline FLAG4-65 & $240 / 286$ & - & $180 / 188$ & - & $264 / 264$ & - & $160 / 198$ & $158 / 194$ & $182 / 224$ & $166 / 206$ & $154 / 190$ & $164 / 202$ \\
\hline FLAG4-71 & $197 / 211$ & - & $185 / 195$ & - & - & - & $187 / 205$ & - & - & $211 / 227$ & - & - \\
\hline FLAG5-83 & $314 / 314$ & - & - & - & - & - & - & - & - & - & - & - \\
\hline FLAG5-87 & $167 / 177$ & - & - & - & - & - & - & - & - & $189 / 189$ & - & $187 / 199$ \\
\hline FLAG6-14 & $304 / 324$ & - & $273 / 277$ & - & $256 / 266$ & - & $228 / 244$ & - & - & - & $264 / 282$ & - \\
\hline FLAG6-77 & $196 / 220$ & - & $148 / 194$ & - & - & - & $124 / 160$ & - & $168 / 176$ & - & $120 / 156$ & $126 / 164$ \\
\hline FLAG7-17 & $178 / 180$ & $168 / 168$ & $172 / 180$ & $178 / 190$ & $172 / 174$ & $176 / 176$ & $176 / 176$ & $174 / 182$ & $176 / 180$ & - & - & $168 / 174$ \\
\hline FLAG8-19 & $171 / 183$ & $190 / 205$ & $187 / 195$ & - & - & $167 / 199$ & - & $193 / 197$ & $185 / 190$ & $181 / 185$ & $167 / 185$ & $179 / 183$ \\
\hline FLAG8-37 & $235 / 237$ & - & - & $202 / 202$ & - & - & - & - & - & - & - & - \\
\hline FLAG8-41 & $239 / 239$ & - & $237 / 237$ & - & - & - & - & - & $241 / 248$ & $221 / 221$ & $245 / 248$ & $253 / 267$ \\
\hline FLAG8-89 & $195 / 209$ & - & $207 / 225$ & 195/195 & - & $221 / 221$ & $219 / 219$ & - & $171 / 183$ & $185 / 197$ & $181 / 189$ & - \\
\hline
\end{tabular}

internal size standard (GeneScan LIZ-600). Twenty-eight primer pairs produced polymorphic bands at the expected sizes. Allele sizes were scored with GeneMapper Software 4.0. Genotypes were analysed with Genetix (Belkhir et al. 1996-2004) and GenePop version 4.0 (Rousset 2008), where polymorphism varied from 8 to 38 alleles with an average of 16 alleles per locus per population (Table 1). Observed heterozygosity ranged from 0.542 to 1 , and only one marker (FLAC4-60) significantly deviated from HardyWeinberg expectations in the Tyne sample after Bonferroni correction. FLAC2-18 was similar to Sciaenops ocellatus microsatellite Scoc76 (GenBank Accession no. EU727070.1). Markers FLAC1-32 and FLAG2-76 appear to be in linkage disequilibrium in both populations and overall, suggesting that both markers are gametically linked. Cross-species amplification on 11 European flatfish species was tested using the same parameters as for flounder (Table 2).

These novel nuclear markers will contribute to the library of available markers and will provide tools to enhance our understanding of the genetic structure of flounder throughout its range, and increase the power of detecting signals of evolutionary processes imposed on populations under contaminant and other environmental stress.

\section{Acknowledgements}

We are thankful to Freya Goodsir for collecting the other flatfish species samples. Niklas Tysklind was supported by a CEFAS-Bangor University joint studentship. Teresa Neuparth was supported by 'Fundação para a Ciência e Tecnologia' fellowship no. SFRH/ BPD/18192/04. This work was supported by Department for Environment Food and Rural Affairs (Defra): Grant no. ME3206

\section{References}

Belkhir K, Borsa P, Chikki L, Raufaste N, Bonhomme F (19962004) Genetix 4.05, Logiciel sous Windows pour la Genetique des Populations. Laboratoire Genome, Populations, Interactions: CNRS UMR 5000, Universite de Montpellier II, Montpellier, France.

Casas L, Martínez P, Sánchez L (2005) Characterization of microsatellite markers derived from sequence databases for the European flounder (Platichthys flesus). Molecular Ecology Notes, 5, 664-665. 
Hall TA (1999) BioEdit: a user-friendly biological sequence alignment editors and analysis program for Windows 95/98/NT. (http://www.mbio.ncsu.edu/BioEdit/bioedit.html). Nucleic Acids Symposium Series, 41, 95-98.

Hemmer-Hansen J, Nielsen EG, Grønkjær P, Loeschcke V (2007) Evolutionary mechanisms shaping the genetic population structure of marine fishes; lessons from the European flounder (Platichthys flesus L.). Molecular Ecology, 16, 3104-3118.

Marchand J, Tanguy A, Charrier G, Quiniou L, Plee-Gauthier E, Laroche J (2006) Molecular identification and expression of differentially regulated genes of the European flounder, Platichthys flesus, submitted to pesticide exposure. Marine Biotechnology, $\mathbf{8}$, 275-294.

Rousset F (2008) GenePop'007: a complete re-implementation of the GenePop software for Windows and Linux. Molecular Ecology Resources, 8, 103-106.

Rozen S, Skaletsky HJ (2000) Primer 3 on the WWW for general users and for biologist programmers. (http://frodo.wi.mit.edu/ cgi-bin/primer3/primer3_www.cgi). In: Bioinformatics Methods and Protocols: Methods in Molecular Biology (eds Krawetz S, Misener S), pp. 365-386. Humana Press, Totowa, New Jersey.

Schuelke M (2000) An economic method for the fluorescent labeling of PCR fragments. Nature Biotechnology, 18, 233-234.

Stentiford GD, Longshaw M, Lyons BP, Jones G, Green M, Feist SW (2003) Histopathological biomarkers in estuarine fish species for the assessment of biological effects of contaminants. Marine Environmental Research, 55, 137-159.

Tysklind N, Taylor MI, Lyons BP, McCarthy ID, Carvalho GR (2009) Development of 30 microsatellite markers for dab (Limanda limanda L.): a key UK biomonitoring species. Molecular Ecology Resources, doi: 10.1111/j.1755-0998.2008.02513.x

Williams TD, Diab A, Ortega F et al. (2008) Transcriptomic responses of European flounder (Platichthys flesus) to model toxicants. Aquatic Toxicology, 90, 83-91. 